\title{
New functionalities developed in the NERIS-TP project regarding meteorological data used by Decision Support Systems
}

\author{
S. Andronopoulos ${ }^{1}$, I. Ievdin ${ }^{2}$, I. Kovalets ${ }^{3}$, S. Anulich ${ }^{3}$ and D. Trybushnyi ${ }^{4}$ \\ 1 NCSR Demokritos, Institute of Nuclear and Radiological Sciences and Technology, Energy and Safety, Aghia Paraskevi, Attiki, Greece. \\ 2 Ukranian Centre for Environmental and Water Projects (UCEWP), prosp. Glushkova, 42, Kiev, Ukraine. \\ 3 Institute of Mathematical Machines \& Systems Problems NAS of Ukraine (IMMSP), prosp. Glushkova, 42, Kiev, Ukraine. \\ ${ }^{4}$ Karlsruher Institut für Technologie (KIT), Campus Nord, IKET, Bau 433, 76021 Karlsruhe, Germany.
}

\begin{abstract}
In this paper a description is given of software tools that have been developed during the NERIS-TP project which provide the capability to users of Decision Support Systems (DSSs), such as JRODOS, to calculate their own prognostic meteorological data with the desired spatial and temporal resolution. These tools increase the flexibility of applying the DSSs for any location on the Earth. This is achieved by downloading freely available global meteorological data and downscaling them using the prognostic meteorological model WRF. The results of WRF and/or global data are uploaded to the DSS to calculate the atmospheric dispersion. The above software tools operate in an automated way, in conjunction with the DSS. In addition, data assimilation methodologies have been integrated into the Meteorological Pre-Processor of JRODOS, to correct previously calculated prognostic data on the basis of locally measured meteorological data. These data assimilation methods were successfully tested and their results increase the accuracy of the prognoses of dispersion models.
\end{abstract}

Keywords: Decision Support Systems / JRODOS / prognostic meteorological data / data assimilation / NERIS-TP

\section{Introduction}

Decision Support Systems (DSSs) for nuclear emergencies, such as JRODOS, need Numerical Weather Prediction (NWP) data to calculate prognoses of dispersion and deposition of radionuclides. Such NWP data are usually provided to the centres operating the DSSs by Meteorological Offices on the basis of bi-lateral agreements for fixed, pre-determined geographical areas. In addition, DSSs use measured meteorological data for calculating nowcasts (diagnoses) or to "correct" previously produced forecasts. The measured data come from on-site stations at the Nuclear Power Plant (NPP) or from a wider-range monitoring network.

One of the goals of the NERIS-TP project was to increase the flexibility of use of DSSs for any location on the Earth, by developing capabilities to calculate "in-house" (i.e., independently of Meteorological Offices) NWP data for the desired computational domain at fine spatial and temporal resolution. The way to achieve this is to use freely available global meteorological data and downscale them to the desired computational domain and fine spatial resolution by running a meso-scale prognostic meteorological model. The above calculations must be connected as much as possible in an automated way to the operation of the DSSs, where the results of the meso-scale model should be uploaded to be used for dispersion calculations.

In NERIS-TP we selected, as freely available global meteorological data, those of the NWP model GFS (Global Forecasting System) operated by the US National Centers for Environmental Protection (NCEP). These data can be downloaded from the NOMADS (National Operational Model Archive \& Distribution System) servers (Rutledge et al., 2006). The model selected for downscaling is the Weather Research and Forecasting (WRF) model (Skamarock et al., 2008) which is freely available to install and run, uses as initial and boundary conditions the above-mentioned global data, and is supported by a very large users' and developers' community. In the NERIS-TP project, a series of software tools have been developed that allow the automatic downloading of global meteorological data, automatic running of WRF for the domain of interest and uploading of either the WRF results or the global data to the Prognosis Database of JRODOS DSS. The interconnections of these software tools are shown in Figure 1, where the particular tools described in this paper are underlined. Furthermore, Data Assimilation (DA) capabilities have been integrated into the Meteorological Pre-Processor (MPP) of JRODOS to allow correction of the forecast meteorological 


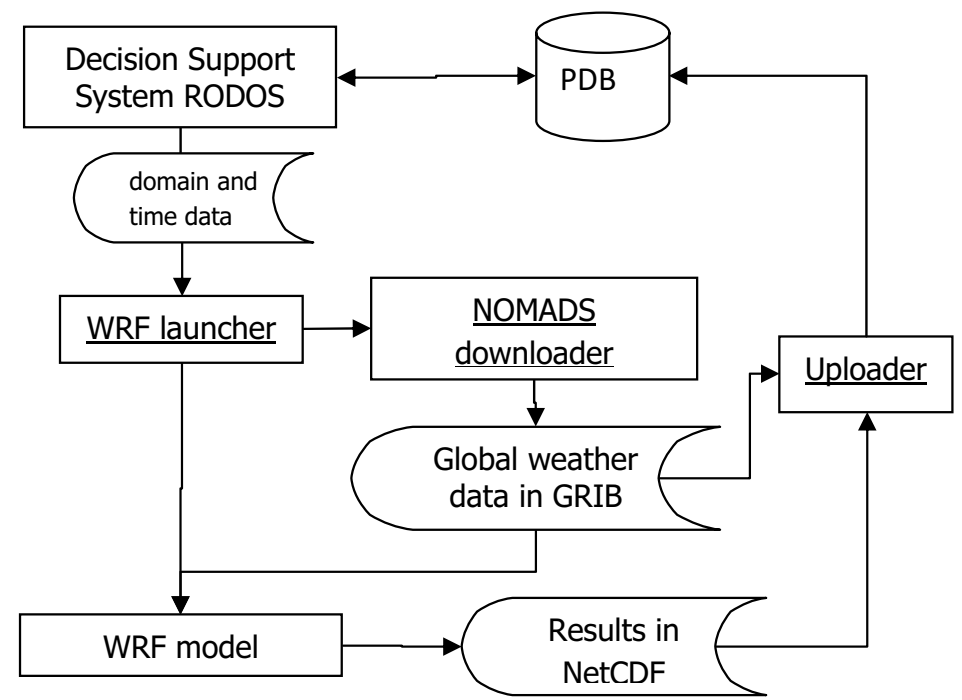

Figure 1. Interconnections of software tools; the tools described in this paper are underlined.

data, taking into account local measurements. In the following sections the above software tools are briefly described and some indicative computational results are presented.

\section{2 "WRF Launcher" and "NOMADS Downloader"}

These software tools (Didkivska et al., 2013) have the purpose of automatically running the WRF meteorological model to provide the JRODOS system with numerical weather prediction data for an arbitrarily located computational domain. The tools can be invoked either from inside JRODOS or externally by the user. In all cases, the triggering message is in the same format $(\mathrm{xml})$ and includes the coordinates of the computational domain's centre (release location), the radius of the domain, start date/time and length of the requested forecast, and a flag indicating whether WRF computations are to be performed or the global data will just be uploaded directly to the JRODOS database. Additional input information for the tool concerns the configuration of the nested computational domains used by WRF. Based on the above information, the WRF Launcher writes the files with the input data needed by WRF. Next, the WRF Launcher invokes the "NOMADS Downloader", which downloads the necessary NCEP global final analysis and / or forecast meteorological data from the NOMADS servers (http://nomads.ncdc.noaa.gov/). Finally, WRF performs calculations using the downloaded global meteorological data as initial and boundary conditions.

\section{Uploader of global and WRF weather data to the DSS}

This software tool (Ievdin and Kovalets, 2012) processes meteorological data in GRIB1 (http://rda.ucar.edu/docs/ formats/grib/gribdoc/) or NETCDF formats and uploads them to the "Prognosis Database" (PDB) of the RODOS system (see Figure 1). So, both the results of the WRF model, which are in
NETCDF format, and the data from most weather prediction centres, which are in GRIB format, can be directly uploaded to the PDB of RODOS to be used for dispersion calculations. The RODOS system itself is not involved in the process of parsing the incoming files, or preparing the data in the appropriate format and uploading the data to PDB, so it is not slowed down during this procedure. The purpose of being able to upload both global data - which have a coarse spatial resolution - and WRF results - which have a fine spatial resolution - to the $\mathrm{PDB}$ is to allow for performing quick dispersion calculations based on the coarse resolution data, while waiting for the more detailed WRF results.

\section{Data assimilation in the meteorological pre-processor}

Data Assimilation (DA) algorithms, allowing the simultaneous use of NWP with measurement data, have been integrated into the operational version of the JRODOS Meteorological Pre-Processor (Kovalets et al., 2014) as part of a Local-Scale Model Chain (LSMC). DA algorithms improve the agreement of the meteorological fields calculated by the MPP with the local-scale measurements, thus increasing the accuracy of the dispersion models' predictions. The following steps are carried out for the calculation of meteorological variables by the MPP including DA procedures (MPP-DA):

1. calculation of the "first guess" wind field, temperature and other meteorological variables on the computational grid of the MPP by spatial interpolation of the corresponding values from the grid of the NWP model;

2. assimilation of the measurements of scalar variables (surface temperature, cloud cover, net radiation, precipitation);

3 . assimilation of the wind velocity measurements;

4. correction of the resulting wind field with a divergenceminimising procedure;

5. final calculation of all remaining meteorological fields, such as the stability category, Monin Obukhov length, 

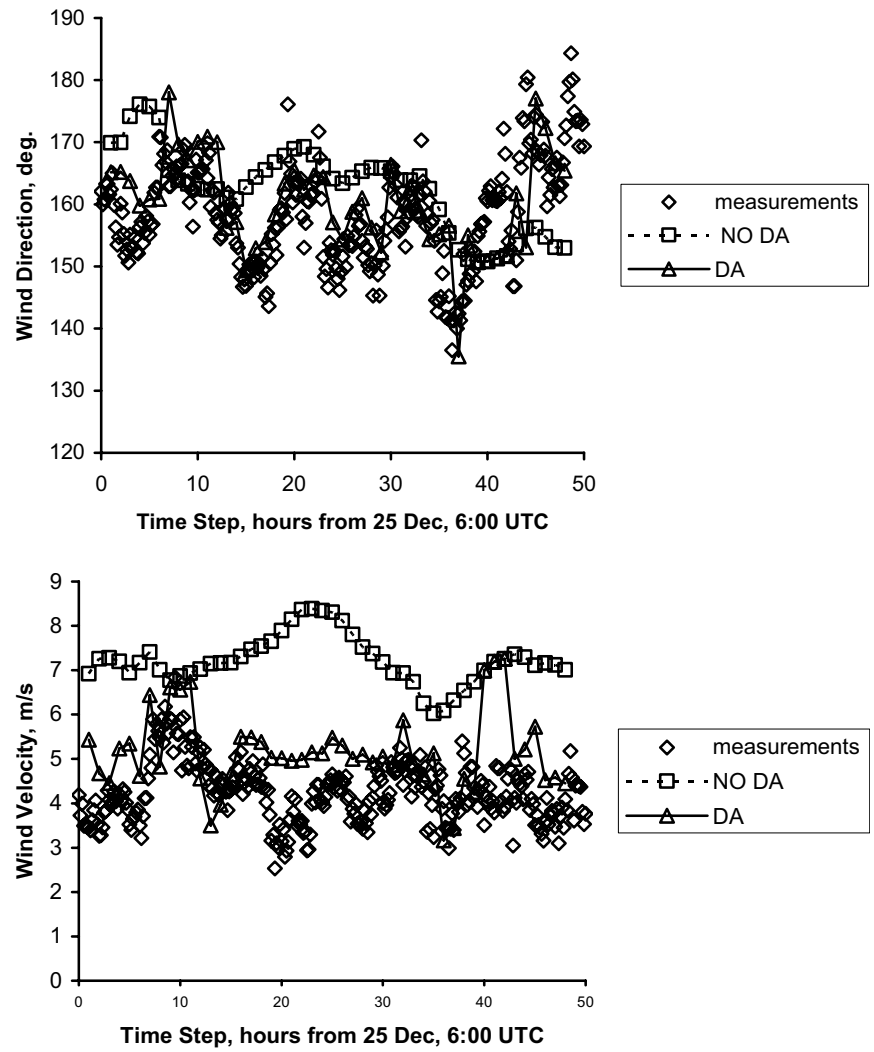

Figure 2. Time dependency of 10-m wind direction and wind velocity as reported by measurements and calculated by JRODOS MPP at the location of the measurement station with and without data assimilation.

friction velocity, etc., based on the existing methodologies in the current version of the MPP of the RODOS system.

Assimilation of the scalar fields' measurements is performed using a variant of the Cressman-type objective analysis procedure called "Iterations to optimal solution" (IOS). Wind velocity observations are firstly interpolated or extrapolated from the observational levels to the MPP grid levels and then they are assimilated through either the IOS method or a multivariate optimal interpolation (OI) method.

The option to activate MPP-DA is set by the user and DA is performed at a certain time step if measurement data are found for this time step. The JRODOS User Interface has been extended to accommodate the new input information related to the functioning of MPP-DA (activating the procedure and setting a user's options). Also, the Project's Result Tree of JRODOS has been extended to include information about MPPDA, and a special output file is also written containing detailed information.

The correctness of MPP-DA integration in the JRODOS System was tested using NWP data calculated with the WRF mesoscale meteorological model and measurements collected near Rivne Nuclear Power Plant (RNPP) from 25-26 Dec 2012. The available measurements included 10 -minute averaged values of the 10-m wind speed and direction, 2-m temperature and humidity, net radiation, precipitation and pressure. Vertical profiles of the wind speed, wind
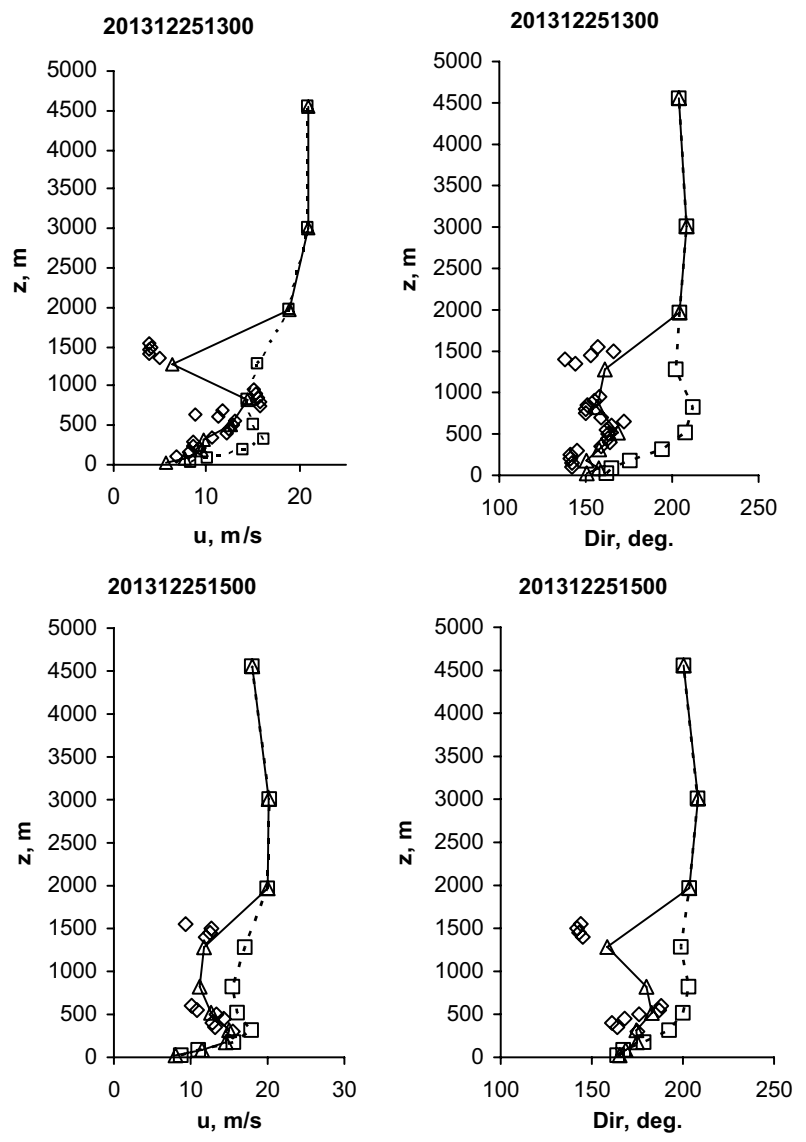

Figure 3. Vertical profiles of wind velocity and wind direction measured by SODAR and calculated by JRODOS MPP with and without data assimilation. Symbols are the same as in Figure 2.

direction and temperature measured by SODAR were also available at heights starting from $100 \mathrm{~m}$ up to 500 or $2000 \mathrm{~m}$. JRODOS MPP was run with and without data assimilation and its results were compared with the corresponding measurements (Figures 2 and 3). It is apparent that in all cases the results obtained with data assimilation are nudged to measurements, indicating a successful implementation. Furthermore, it is obvious that data assimilation has a significant effect on the corresponding calculated concentration fields by the JRODOS atmospheric dispersion models, as can be seen in Figure 4.

\section{Conclusions}

In the NERIS-TP Project, software tools have been developed that promote the independence of operators of Decision Support Systems regarding the provision of prognostic meteorological data and increase the flexibility of using the DSS for any location on the Earth with the desired spatial and temporal resolution. In addition, Data Assimilation (DA) algorithms, allowing the simultaneous use of NWP with measurement data, have been integrated into the operational version of the JRODOS Meteorological Pre-Processor. Meteorological DA increases the accuracy of the dispersion models' predictions. It is concluded that it can be very important to use the developed algorithms of meteorological data assimilation in combination 


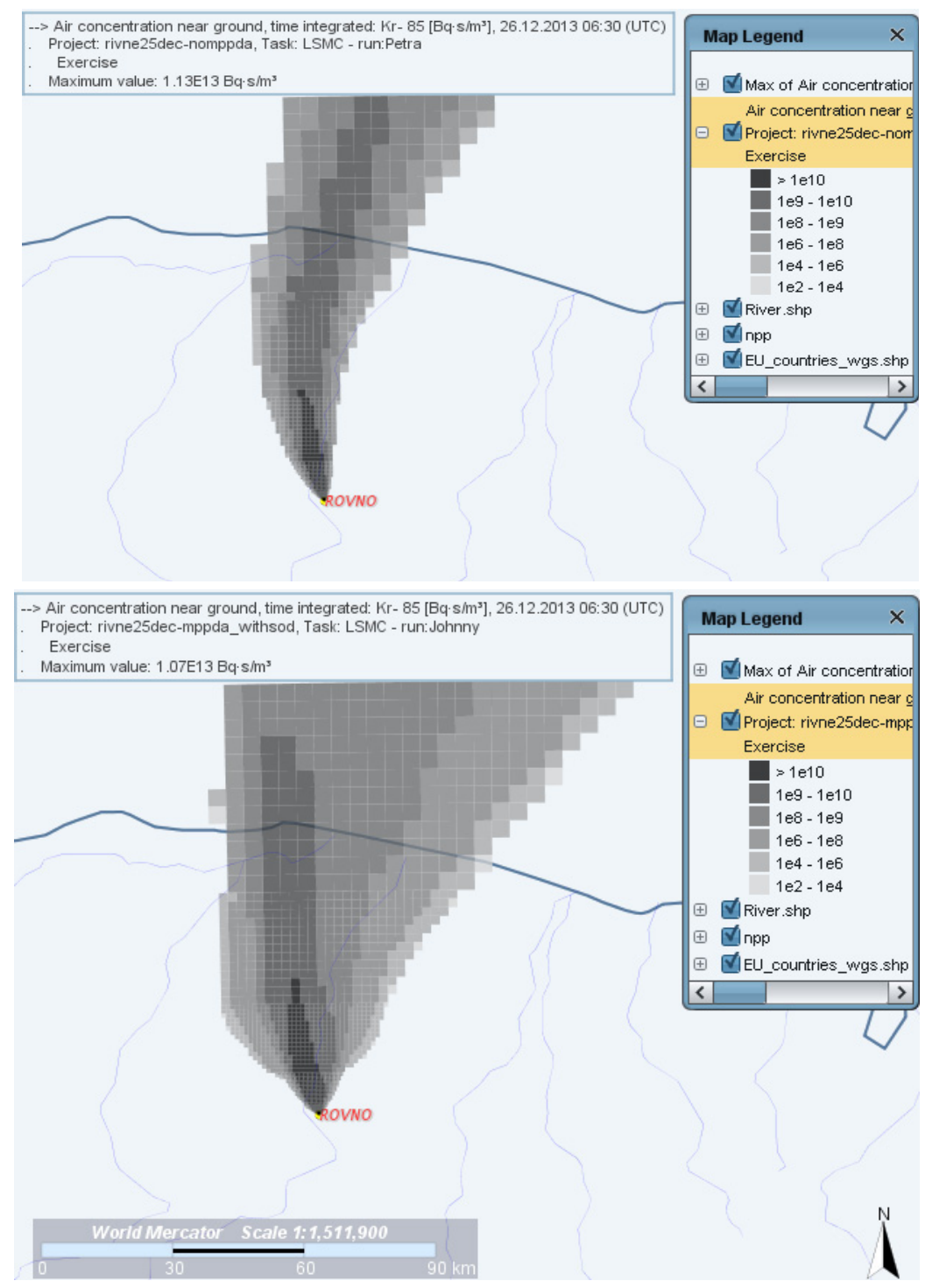

Figure 4. Time-integrated concentration distributions calculated by JRODOS RIMPUFF over 24 hours following stationary release of ${ }^{85} \mathrm{Kr}$ $\left(5 \times 10^{17} \mathrm{~Bq} / 24\right.$ hours) starting at 6:30 UTC, $25 \mathrm{Dec}$, without data assimilation (upper) and with data assimilation (bottom) in the Meteorological Pre-Processor.

with source inversion methods, which are currently underdeveloped for the JRODOS System and will be implemented in the near future.

\section{References}

Didkivska S., Ievdin I., Kovalets I., Trybushnyi D. (2013) Software tools and related documentation for the automatic downloading of global meteorological data and operation of the WRF meteorological modelling system, Report NERIS-TP(WP4)-(13)-02.

Ievdin Y., Kovalets I. (2012) Uploader of global and WRF weather data to the DSS in GRIB1 format Technical Description and User Guide, Report NERIS-TP(WP4)-(12)-01.
Kovalets I., Ievdin I., Andronopoulos S. (2014) Integration of data assimilation algorithms in the meteorological pre-processor of the JRODOS System, Report NERIS-TP(WP4)-(14)-02.

Rutledge G.K., Alpert J., Ebisuzaki W. (2006) NOMADS - a Climate and Weather Model Archive at the National Oceanic and Atmospheric Administration, Bulletin of the American Meteorological Society 87, 327-341.

Skamarock W.C., Klemp J.B., Dudhia J., Gill D.O., Barker D.M., Duda M.G., Huang X.Y., Wang W., Powers J.G. (2008) A description of the advanced research WRF version 3. NCAR Technical Note NCAR/TN-475+STR, Boulder, Colorado, USA, 125 p., http://www.mmm.ucar.edu/wrf/users/docs/arw_v3.pdf. 